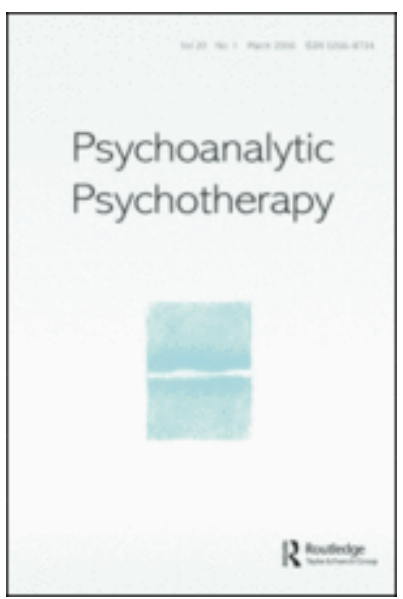

Short Term Psychodynamic Psychotherapy (STPP) for Clients with Complex and Enduring Difficulties within NHS Mental Health Services: A Case Series

\begin{tabular}{|r|l|}
\hline Journal: & Psychoanalytic Psychotherapy \\
\hline Manuscript ID & RPPS-2020-0013.R1 \\
\hline Manuscript Type: & Original Article \\
\hline Keywords: & Short-Term, Psychodynamic, Psychotherapy, NHS, Secondary, Care \\
\hline \multicolumn{2}{|l}{} \\
\hline
\end{tabular}




\section{Short Term Psychodynamic Psychotherapy (STPP) for Clients with Complex and Enduring Difficulties within NHS Mental Health Services: A Case Series}

Word count: 4,388 (without abstract, references \& tables) 
Short Term Psychodynamic Psychotherapy in the NHS

\begin{abstract}
Purpose: Short Term Psychodynamic Psychotherapy (STPP) has been demonstrated as an effective treatment for several mental health difficulties. However, its implementation in secondary mental health services in the National Health Service (NHS) is scarce. The aim of this study was to bridge the gap between controlled trials and practice-based evidence, by exploring an initial estimate of the therapeutic effects of this intervention in a secondary care NHS community mental health setting. Method: The study followed a quantitative case series design. Eight clients with complex, enduring mental health difficulties, supported by a community secondary mental health service received a course of STPP. They completed outcome measures at the start, at the end and eight-weeks following completion of therapy. Results: All participants but one completed the therapy and attendance rates were high (>75\%). No adverse effects were reported. All participants but two reported improvement in the CORE-OM, BSI and the PHQ-9 and these were maintained at follow-up. Conclusions: The results suggested that STPP was a safe and acceptable intervention, that may have contributed to clinical and reliable improvement for 4 participants, non-reliable improvement for 2 and non-reliable deterioration for one participant who finished the treatment.
\end{abstract}


Short Term Psychodynamic Psychotherapy (STPP) is an alternative to original long term, intensive and often expensive psychoanalytic treatments. STPP formal models were not established until the 1970s and some of its main theoretical contributors include Balint (1972), Bowlby (1973), Davanloo (1980), Malan (1979) and Ferenzi (1925) amongst others. Although there are several variants of STPP, they are all concerned with unconscious impulses, have a focus on emotional experiences, and place great emphasis on the client's attachment/interpersonal styles as represented in their personal life and in the therapeutic relationship. Thus, STPP can be considered as an umbrella term that encompasses psychodynamic/psychoanalytic therapies that have common processes: mainly being timelimited, maintaining a therapeutic focus (as opposed to free association in open ended psychoanalysis), re-structuring of defences, anxiety regulation and facilitation of the expression of affect (Kenny, 2016).

\section{Empirical Evidence of STPP}

There are currently over 250 published randomised controlled trials (Liliengren, 2020) supporting the use of STPP with several mental health presentations including personality and somatic disorders, anxiety, post-traumatic stress disorder (PTSD) and depression among others (Abbass et al., 2014). In fact, several meta-analyses examining the efficacy of STPP have shown large effect sizes that persist at follow-up (Abbass \& Driessen, 2010; Abbass, Kisley, \& Kroenke, 2009; Abbass, Town, \& Driessen, 2011; Driessen, et al., 2015; Leichsenring, Rabung, \& Leibing, 2004; Liliengren et al., 2016; Town, Abbass, \& Hardy, 2011). Furthermore, STPP is included in the NICE guidelines for depression (NICE, 2018) and eating disorders (NICE, 2017). 


\section{Habib Davanloo's STPP Developments}

Davanloo's treatment model is a short psychodynamic therapy of integrative nature, which includes elements from attachment, modern psychodynamic theories and the neurobiology of anxiety processes (Abbass \& Schubiner, 2018). In this treatment model, the triangle of conflict (see figure 1; Ezriel,1952; Malan,1979) is used in order to help clients to process complex emotional experiences that are outside of their conscious awareness. The STPP therapist adopts a relational approach of enquiry and attempts to help the client to understand the triangle and how the difficulties are being perpetuated within it. Clinicians also use Malan's triangle to help the client explore how emotions generated in early attachment relationships (with caregivers), can often become triggered in current relationships (i.e. with partners) and also in the transference relationship with the therapist.

\section{Insert Figure 1}

The treatment includes active interventions such as anxiety regulation, challenge and confrontation of defences as well as exploration of transference feelings and how they are linked to past and current relationships. Davanloo's model of therapy begins with a specialised assessment interview called "trial therapy" which lasts between 2 and 4 hours, and can be undertaken in one or more sessions (Davanloo, 2005; Ten Have-De Labije \& Neborsky, 2012). During this trial therapy, specific therapeutic techniques are used to engage the client and assess their responses to these techniques. Whether the responses are positive or not, is used as the primary inclusion criterion, rather than a specific psychiatric diagnosis (Davanloo, 2005). A more detailed description of the treatment methods and techniques of STPP can be found in various papers outlining key clinical processes of the treatment model, such as Abbass and Town (2013); Ten Have-De Labije and Neborsky (2012). 
This therapy has been designed to help address interpersonal and intrapsychic conflicts; something advantageous for clients who receive support from secondary mental health services in the NHS who often do not fit in a single diagnostic category but experience significant difficulties in interpersonal domains. Davanloo's transdiagnostic treatment model has yielded promising results, showing improvements in symptomatology for clients diagnosed with depression, somatic, anxiety and personality disorders, with effect sizes (Cohen's d) ranging from .84 (interpersonal problems) to 1.51 (depression; Abbass, Town and Driessen, 2012).

\section{Current Study}

A few pilot studies have implemented STPP treatments in NHS settings such as primary care (Lemma, Target \& Fonagy 2011), Tier 4 (Haikowski \& Buller, 2012) or specialist services (Russell, 2017). Nevertheless, there is a lack of 'on the ground' practice-based outcome studies of psychodynamic psychotherapy within NHS services (Ward, Wood \& Awal, 2013), in particular within community mental health teams (CMHT).

Therefore, the primary aim of this study was to explore whether there were changes in psychological distress, mood and mental health symptomatology following STPP, with patients with different mental health presentations in a naturalistic clinical setting. The secondary aim of this study was to explore the acceptability and safety of STPP. The initial hypotheses were that all participants would show symptomatic improvements that would be maintained at follow-up and that the intervention would be safe and acceptable in a secondary care NHS service.

\section{Method}

\section{Pre-registration}


The original study protocol and proposal for this case series including study aims, method and design were pre-registered in October 2019 on the Open Science Framework (Link not provided to avoid revealing authors) in order to prevent selective reporting bias. Changes, additions and minor deviations from the original protocol are described in Appendix 1. The protocol was submitted for ethics approval prior to the start of the study and was anonymised to prevent identification of the service. Ethical approval was granted by the Research and Development (R\&D) department of the NHS Trust where the study was conducted.

\section{Study Design}

Psychological therapeutic intervention case-series.

\section{Participants}

A total of eight participants provided informed written consent to take part in this study, which was advertised as a service evaluation using a case series design. In order to meet inclusion criteria, participants were 18 years or older, currently under the care of the NHS adult secondary mental health team, and considered capable of providing informed consent via the lead clinician or the clinical team. All participants were required to have been offered STPP, informed of its purpose/methods and agreed to engage with this therapeutic model.

The exclusion criteria included the presence of an identified comorbid intellectual disability or autistic spectrum condition as determined by the individual's clinical team. Individuals experiencing a current ongoing psychotic or manic episode, and those who were receiving any other forms of formal psychological intervention were also excluded from the study. 


\section{Procedure}

Participants who were on the waiting list for a psychological intervention within the service were informed about the possibility of taking part in the study. Initially, participants entered the trial therapy phase and a decision of whether STPP was appropriate was then made collaboratively after 2-4 sessions. This is consistent routine clinical practice within this therapeutic model. Participants who were already receiving a course of STPP by the lead author were also invited to take part in the study. Nine participants were invited to take part in the study and eight consented. Of these eight, four were already receiving a STPP intervention and the remaining four were invited from the psychology waiting list. Participants were considered to be enrolled in the study if they had completed the participation consent form and were attending STPP therapy sessions. Participants were made aware that whilst the study was running, they could withdraw at any time and have their data destroyed without any consequence for their treatment.

All participants were invited to complete several outcome measures in face-to-face meetings at the beginning and end of therapy (see measure details below). The eight-week follow-up outcome measures were posted to participants' home addresses and were returned by post. A follow-up call was conducted if participants had not returned the questionnaires within 10 days. All participants who entered the trial therapy $(\mathrm{N}=8)$ participated in the study. Sociodemographic and clinical information was extracted from the medical records of the participants with their written consent. As part of the standard clinical practice of the lead clinician for training in STPP, informed consent for video-recording therapy sessions was obtained from four participants.

\section{Therapy}

STPP was offered as part of participants' usual care within the NHS service where this study took place. This meant that the therapy was representative of a STPP intervention 
within the NHS. However, this resulted in heterogeneity in the session content, number and timings of sessions provided. Although the length of STPP varies, research often describes between 7 and 40 sessions (Leichsenring, Rabung \& Leibing, 2004; Markowitz, Svartberg \& Swartz, 1998). The length of treatment offered in this study was dependent on clinical judgement for each participant and was consistent with the length of therapy usually offered in the service which ranges between 16-24 sessions. This variability in the treatment is consistent with Medical Research Council guidance for case series design (Craig et al., 2008).

Therapy involved weekly 50 minutes session and the trial therapy was conceptualised as the initial three sessions. Therapy adhered to Davanloo's model of STPP including: an initial focus and understanding of attachment patterns, specific assessment and regulation of anxiety pathways, identification of defensive manoeuvres, development of a collaborative understanding of the triangle of conflict and the triangle of person, focusing on helping the patient's awareness of self-destructive behaviours, a focus on emotional processing, attendance to transference feelings and ongoing monitoring and discussion of the ending of therapy.

The therapy was delivered by the lead author, as the only member of staff with formal training in this therapeutic model. He has over 2 years of postgraduate training in this therapeutic modality and received monthly supervision from accredited psychodynamic therapists during the study. The therapist competence was assessed through monthly monitoring of video recordings of sessions, which was consented to by participants $(\mathrm{N}=4)$.

\section{Instruments}

\section{Primary Outcomes}




\section{The Clinical Outcomes in Routine Evaluation Outcome Measure (CORE-OM).}

This is a 34-item self-report questionnaire designed to be administered before and after therapy (Evans et al., 2000). The respondent uses a 5-point Likert scale to indicate the extent to which each of the 34 statements reflects how they have been over the last week, ranging from 0 (not at all) to 4 (most or all of the time). The 34 items cover four dimensions including subjective wellbeing, symptoms, life functioning and risk to self/others. The responses are then averaged to produce a mean score, which indicates the level of psychological distress (raging from 'healthy' to 'severe'). The clinical score is then obtained by multiplying this mean score by ten, resulting in scores between 0 and 40 .

Initial findings suggest that the measure is easy to read, and acceptable to both native and non-native English speakers. Evans et al. (2002) found appropriate internal reliability ( $\alpha$ of between .75 and .95 ) for both clinical and non-clinical samples on all domains in the CORE-OM. Strong one-week test-retest reliability was found in a small sample of students, ranging from .87 to .91 for all domains, other than the 'risk' domain, which was .64 .

Furthermore, they found good convergent validity with existing measures and clinician ratings of risk, and large significant differences between scores of clinical and non-clinical samples. This along with its free availability have resulted in its widespread use.

Brief Symptom Inventory (BSI). The BSI is the short version of the SCL-R-90 (Derogatis, 1977). This self-report questionnaire consists of 53 items and covers nine different dimensions: Somatization, Obsession-Compulsion, Interpersonal Sensitivity, Depression, Anxiety, Hostility, Phobic anxiety, Paranoid ideation and Psychoticism. All items are rated on a 5-point Likert scale from 0 (not at all) to 4 (extremely). There are also three global indices: Positive Symptom Distress Index, Positive Symptom Total, and Global Severity Index. The present study only includes the Global Severity Index (GSI) score from 
this measure, as this composite mean score combines information about the number of symptoms and intensity of distress. Acceptable internal consistency has been shown on this measure, ranging from .71 on the Psychoticism dimension to .85 on the Depression dimension. The internal consistency for GSI has been reported as .90 (Derogatis \& Spencer, 1982). Construct validity was found to be sufficient, as were convergent and discriminant validity; when compared to the Minnesota Multiphasic Personality Inventory, correlation coefficients ranged from .31 to .72 (Derogatis, 1993).

Patient Health Questionnaire (PHQ-9). The PHQ-9 (Spitzer, Kroenke \& Williams, 1999) is a nine item self-report questionnaire validated in primary care for evaluating a client's mood over the previous two weeks prior to assessment. The measure is based on the diagnostic criteria for major depressive disorders in the Diagnostic and Statistical Manual Fourth Edition (DSM-IV; American Psychiatric Association, 2000). Scores range from 0 to 27, with cut-off scores of 5, 10, 15, and 20 representing 'mild', 'moderate', 'moderately severe' and 'severe' symptoms of depression, respectively. This tool is used to monitor both the severity of depressive symptoms and response to treatment, and has been found to have good criterion validity, and reliability (Kroenke, Spitzer, Williams, \& Löwe 2010). Criterion validity has been found to range between .77 and .88 for sensitivity, and from .88 to .94 for specificity in various studies (Gilbody, Richards, Brealey, \& Hewitt, 2007; Kroenke, Spitzer, \& Williams, 2001; Wittkampf, Naeije, Schene, Huyser, \& van Weert, 2007). Internal validity has been found to range from .86 to .89 , and test-retest reliability was shown to be .84 (Kroenke et al., 2001).

Clinical and Sociodemographic Information. Additional demographic information including the participants' age, gender, ethnicity, relationship status, education, and employment status were extracted from medical records. Information was also gathered 
regarding diagnosis, medication, previous suicide attempts and current self-harm, along with information regarding length of time under secondary mental health services and previous therapeutic intervention.

\section{Secondary Outcomes: Acceptability and Safety}

The acceptability of STPP was judged based on whether (a) $75 \%$ of the sample reached the end of trial therapy (normally completed around session 3-4), and/or (b) $40 \%$ of the sample completed the STPP therapy (criteria adapted from Taylor et al., 2018). These criteria were established a priori as indicated in the pre-registration protocol.

Safety of STPP was assessed through the completion of the Adverse Experiences in Psychotherapy (AEP; Hutton, Byrne \& Morrison, 2017: unpublished) questionnaire. This 28item questionnaire asked participants about a variety of possible adverse experiences that may occur in therapy (such as, "Taking part made me think too much about bad things that have happened in the past"). Mirroring the approach adopted in a CAT case series within NHS secondary services for psychosis (Taylor et al., 2018), items with a rating >3 ('a little') were considered problematic. Furthermore, the lead clinician also monitored the occurrence of serious adverse events. Following Taylor et al.'s (2018) approach, serious adverse events were described as including suicide, suicidal attempts, or having serious risk symptomatic exacerbation (clinically significant increases in the risk subscale of the CORE-OM).

\section{Statistical Analyses}

The main demographics and clinical characteristics of the sample were described through descriptive analyses. Reliable change for the CORE-OM and clinically reliable change for the CORE-OM and PHQ-9 were assessed. Rates of reliable change refer to changes in scores that fall beyond the range which could be attributed to the measurement variability of the instruments themselves. Reliable change in the CORE-OM was defined as a 
change of 5 or more points in the total scores (Evans et al., 2002). Reliable clinical change is defined as scores moving from the "dysfunctional" to the "functional range". In the case of the PHQ-9, a cut-off score of less than 10 was employed, to represent the "functional range" (Manea, Gilbody \& Mcmillan, 2012). For the CORE-OM a decrease of 10 points in the clinical score was employed (Evans et al., 2002). All statistical analyses were conducted using IBM SPSS statistics software, version 22.

\section{Results}

\section{Sample Characteristics}

Insert Table 1

Eight participants who were under the care of an NHS secondary care mental health service were included in the study. Their demographic data are presented in Table 1. The age of participants ranged from 26 to 57 years $(M=39.75 ; S D=11.49)$. There were equal numbers of males and females, all described themselves as White British and four reported being married or in a stable relationship. Although one of the participants discontinued therapy after four sessions, all participants completed the pre and post-treatment outcome measures, and four completed measures at 8-weeks follow-up.

\section{Insert Table 2}

All participants described receiving at least one psychological intervention in the past, and the number of years receiving secondary mental health care support ranged from 1 to 13 years $(M=4.13, S D=3.98)$. Seven participants were being prescribed more than one psychiatric drug and four were currently being prescribed antipsychotic medication. Six participants had attempted suicide before the study and one was engaging in self-injurious behaviours at the time of consenting to enter the study (see Table 2). 
Insert Table 3

The average scores for baseline and post-treatment assessments are presented in Table 3. The average CORE-OM total score at the start of therapy was $18.11(\mathrm{SD}=3.77)$, suggesting the presence of "moderate distress" levels. At post-treatment, the average CORE-OM total score was $13.45(\mathrm{SD}=7.33)$ suggesting that the distress levels had reduced to the "mild range" (Evans et al., 2000). Furthermore, the mean difference between baseline and post-STPP CORE-OM scores was 4.7 (range 1 - 17) points, which is close to the 5-point difference considered to be indicative of reliable change. In addition, both the Symptoms (Pre $\mathrm{M}=22.8$, $\mathrm{SD}=8.62$; Post $\mathrm{M}=16.62, \mathrm{SD}=8.42$ ) and the Functioning (Pre $\mathrm{M}=18.12, \mathrm{SD}=2.64$; Post $\mathrm{M}=12.87, \mathrm{SD}=9.16$ ) subscales of the CORE-OM showed changes that were clinically reliable ( $>5$ points) with a reduction of 6.18 and 5.25 points respectively on average. Similarly, the changes in the Wellbeing subscale (Pre $M=24.06, S D=7.89$; Post $M=19.68$, $\mathrm{SD}=10.89$ ) showed a reduction of 4.38 , which is close to that considered clinically reliable. However, the Risk subscale demonstrated a small change not considered clinically reliable (Pre $\mathrm{M}=4.96, \mathrm{SD}=6.2 ;$ Post $\mathrm{M}=4.13, \mathrm{SD}=5.75$ )

Insert Figure $2 \mathrm{a} \& 2 \mathrm{~b}$

Examination of individual scores shows that two participants (IDs 3 and 8) experienced non-reliable ( $<5$ points) increase in the CORE-OM total scores. In contrast, the other six participants' total CORE-OM scores decreased following STPP (see figure 2a). Of those, participant 1 showed a clinically reliable decrease ( $>5$ points) and participants 2 and 7 showed a decrease of 10 points or more. A reduction of over 10 points indicated that following therapy these participants moved from "dysfunctional ranges" to "healthy ranges". Additionally, Participant 4 showed a change moving towards clinical reliability (reduction of 4.2 points at follow-up). These positive changes were maintained at follow-up (see figure $2 \mathrm{~b}$ ). 
Insert Figure 3a \& 3b

Mean reductions in BSI scores were also found following STPP intervention (Pre$\mathrm{M}=1.75, \mathrm{SD}=0.60$ and Post $\mathrm{M}=1.07, \mathrm{SD}=0.61)$, suggesting an overall improvement in symptoms. When individual participant scores were examined, although participant 3 and 8 again did not show improvements (non-reliable score increase of 0.3 and 0.31 points respectively), all other participants showed a decrease in their BSI scores, which was maintained at follow-up (see Figure 3a \& Figure 3b).

Insert Figure $4 a \& 4 b$

Finally, there were also reductions in mean PHQ-9 scores from baseline (M=16.00, $\mathrm{SD}=5.23)$ to post $(\mathrm{M}=9.63, \mathrm{SD}=5.18) \mathrm{STPP}$ measurement. These differences could be considered a clinically reliable change, with the mean score following therapy falling within the 'healthy' range $(<10)$. When considering individual participant's scores, all except two (Participants 3 and 8), showed a decrease in depressive symptomology as assessed by the PHQ-9, which was maintained at follow-up measurement.

\section{Acceptability \& Adherence}

All participants attended at least four sessions (trial therapy) and seven (87\%) completed the full treatment. The participant who did not complete therapy, reported reasons related to lack of time availability to attend sessions. For the seven participants completing therapy, the average attendance rate was $84 \%$ (mean number of sessions $=17.57$, range $=14$ 20 ), whilst for the full sample, it was $76 \%$ (mean number of sessions $=15.8$ range $=4-20$; See Table 2). Although no formal adherence rating to the therapy was employed, seven sessions (4 different participants) were video supervised with accredited psychodynamic psychotherapists. 


\section{Safety}

Two participants received changes to their medication treatment during the study, both relating to sleeping medication. No other changes in medication/usual care of the participants occurred whilst in therapy. No serious adverse events were identified or reported during the study such as hospitalisations, planning of suicide attempts, suicidal behaviours, or increase in self-harming behaviours. This was corroborated by the lack of increases in the CORE-risk subscale post-therapy or at follow-up measurements (See Table 3). Five participants were discharged from secondary care mental health services following the completion of STPP and they did not have any contact with secondary care or emergency services within the eight weeks of the follow-up period.

The endorsement of self-reported adverse experiences in the AEP were minimal. The average scores for each item of the AEP (reported in Appendix 3), ranged from 1 to 2.5; falling below the score of 3 , which corresponds to "a little", in response to how distressing the adverse experience had been, during, post- therapy or at follow-up ( 8 weeks postcompletion of therapy)

\section{Discussion}

Short-Term Psychodynamic Psychotherapy is an evidence-based intervention (Liliengren et al., 2016) with promising potential as a psychological treatment for some service users supported in NHS secondary care settings. Nevertheless, to date, studies evaluating this treatment within NHS settings is limited. This case-series aimed to examine the effects of STPP as well as explore its acceptability and safety within a secondary care mental health context. The participants in this sample had a variety of long-standing mental health difficulties and the sample could be classed under the label "treatment resistant" (Solbakken \& Abbass, 2013). This describes non- 
respondence (in terms of significant symptom improvement) to three or more treatment attempts (psychological or pharmacological) and is supported by the fact that all participants had been under secondary care for several years. Davanloo's STPP model has an explicit focus on handling resistance in treatment through emotional mobilisation in the transference. Emerging evidence shows that this particular therapeutic modality appears as an effective option for 'treatment resistant' patients who have showed little or no response to other forms of treatment (Abbass, 2016; Town et al., 2017; Town et al., 2020).

This appears to be consistent with the results of this case series as the primary hypothesis was partially met, with 6 out of the 7 participants who completed the therapy showing symptomatic improvements and four showing almost reliable or clinically reliable changes. In addition, these changes appeared to have been maintained at follow-up $(\mathrm{N}=4)$. Furthermore, five participants were discharged from secondary care services after therapy following a multidisciplinary team decision and they had not returned to secondary care or crisis services eight weeks after. In addition, the results of this study are also consistent with a pilot study conducted in an NHS secondary care setting by Hajkowski \& Buller (2012), reporting positive pre-post treatment effects with a sample of treatment resistant patients in a complex care service.

Regarding the second hypothesis, attendance rates clearly satisfied our prespecified targets to assess acceptability. All the participants but one, completed the full therapy and all of them completed the trial therapy sessions. Furthermore, the percentage of session attendance was high, compared to non-attendance rates in standard psychiatric 
services (Killaspy, Banerjee, King \& Lloyd, 2000) and similar or greater when compared to similar psychotherapy case series conducted in NHS secondary services (Taylor et al., 2018). In addition, no adverse events were noted and the answers to the AEP supported the safety of this approach within a secondary care setting. Only participant 3 and participant 8 showed slight post-treatment deterioration and these changes were nonreliable. This could be partially be explained by the fact that participant 3 only attended the initial therapy phase ( 4 sessions) and participant 8 had a diagnosis of paranoid personality disorder, which typically responds best to at least 40 sessions of STPP (Ten Have-de Labije, J., \& Neborsky, 2012).

\section{Strengths and Limitations}

Overall, few studies reported in the literature report on treatment resistant samples (Solbakken \& Abbass, 2013). This case series reports outcomes of patients from a naturalistic NHS secondary care setting with complex and chronic difficulties and who have showed limited response to previous mental health treatments. Noteworthy, most of the published literature on Davanloo's treatment model uses expert therapists whereas this paper presents the outcomes of a trainee psychodynamic psychotherapist, increasing the applicability of this therapy by professionals at different stages of training.

However, caution is warranted and there are some limitations to this study. First, the sample size $(\mathrm{N}=8)$ limits the generalisability of the results. Although the sample size was comparable to similar case series (Abbass, 2006; Hinson, Weinstein, Bernard, Leurgans, \& Goetz, 2006; Taylor et al., 2018) and consistent with the objectives of the study, the conclusions of this report are limited to the specific service where this study was conducted. Future studies should aim to include larger samples and greater number of therapists, to expand the generalisability of the results. Furthermore, the sample 
reflected a lack of ethnic diversity as there were no participants from ethnic minority groups. Another selection bias occurred as some of the included participants weere already in treatment when they received the invitation to take part in the study. Third, given that there were no control groups, it is not possible to determine causality in the changes experienced post therapy, as these changes may reflect natural variations of symptoms over time. Future studies should aim to compare the intervention with a waiting list or control group.

Fourth, the high attendance rates suggest participants might have found this therapy approach helpful. However, the data of this study is quantitative thus limiting the understanding of the value of specific STPP tools or techniques from the perspective of the participants. Future papers would benefit from mixed methods design where themes and reflections of the experiences of this therapy model can be gathered. Lastly, there was only one therapist in this study and whilst his practice was supervised through video recordings by STPP accredited supervisors, the lack of a formal rating adherence scale is a limitation regarding adherence to the treatment model. Future research should aim to use a standardised, validated treatment adherence tool/inventory.

\section{Conclusions}

Ultimately, the results suggest that this intervention was safe, acceptable and did not lead to any adverse events or clinical deterioration for the participants. It also suggests that the degree of engagement of participants with the therapy was high and that it might have contributed to a clinical improvement which is reflected by the self-report questionnaires and by the decision of being discharged from secondary care services following a long period of support. However, these data do not present with a clear picture or understanding of the effects of STPP in clinical outcomes for general secondary care NHS clients and this should 
be further addressed in future naturalistic studies with greater samples and comparison groups. 


\section{References}

Abbass, A. (2006). Intensive short-term psychotherapy of treatment-resistant depression: A pilot study. Depression and Anxiety, 23(7), 449-452. https://doi.org/10.1002/da.20203

Abbass, A. (2016). The emergence of psychodynamic psychotherapy for treatment resistant patients: Intensive short-term dynamic psychotherapy. Psychodynamic Psychiatry, 44(2), 245-80. DOI: 10.1521/pdps.2016.44.2.245

Abbass, A. \& Driessen, E. (2010). The efficacy of short-term psychodynamic psychotherapy for depression: A summary of recent findings. Acta Psychiatrica Scandinavica, 121(398); author reply 398-399. doi: 10.1111/j.1600-0447.2009.01526.x.

Abbass, A. \& Katzman, J. (2013). The cost-effectiveness of intensive short-term dynamic psychotherapy. Psychiatric Annals, 43(11), 496-501. DOI:10.3928/00485713-2013110504.

Abbass, A., Kisley, S., \& Kroenke, K. (2009). Short-Term Psychodynamic Psychotherapy for Somatic Disorders. Psychotherapy and Psychosomatics, 78(5), 265-274. doi: $10.1159 / 000228247$

Abbass A. A., Kisely, S. R., Town, J. M., Leichsenring, F., Driessen, E., De Maat, S., . . . \& Crowe, E. (2014). Short-term psychodynamic psychotherapies for common mental disorders. Cochrane Database Systematic Reviews 7:CD004687. DOI: 10.1002/14651858.CD004687.pub4.

Abbass, A. \& Schubiner, H. (2018). Hidden from View: A Clinician's Guide to Physiologic Disorders. (1 $1^{\text {st }}$ ed.). Mind Body Publishing.

Abbass, A. \& Town, J. M. (2013). Key clinical processes in intensive short-term dynamic psychotherapy. Psychotherapy, 50(3), 433-437. Doi:10.1037/a0032166 
Abbass, A., Town, J., \& Driessen, E. (2011). The efficacy of short-term psychodynamic psychotherapy for depressive disorders with comorbid personality disorder. Psychiatry, 74(1), 58-71. doi: 10.1521/psyc.2011.74.1.58.

Abbass, A. A., Town, J. M., \& Driessen, E. (2013). Intensive short-term dynamic psychotherapy: A review of the treatment method and empirical basis. Research in Psychotherapy: Psychopathology, Process and Outcome, 16(1), 6-15. DOI: 10.7411/RP.2013.002

American Psychiatric Association. (2000). Diagnostic and Statistical Manual of Mental Disorders, Fourth Edition - Text Revision (DSMIV-TR). Washington DC: American Psychiatric Press.

Balint, M., Ornstein, P. H., \& Balint, E. (1972). Focal Psychotherapy: An Example of Applied Psychoanalysis. (1 ${ }^{\text {st }}$ ed.). London: Tavistock Publications Limited.

Bowlby, J. (1973). Attachment and loss. Vol. 2: Separation: anxiety and anger. ( $\left.1^{\text {st }} \mathrm{ed}.\right)$. New York, NY: Basic Books.

Craig, P., Diepe, P., Macintyre, S., Michie, S., Nazareth, I., \& Petticrew, M. (2008). Developing and evaluating complex interventions: New guidance. Retrieved from www.mrc.ac.uk/complexinterventionsguidance

Davanloo, H. (1980). Short-term Dynamic Psychotherapy. (1 $1^{\text {st }}$ ed.). New Jersey: Jason Aronson.

Davanloo, H. (1987). Unconscious therapeutic alliance. In P. Buirski (Ed.), In frontiers of dynamic psychotherapy (pp. 64-88). New York: Mazel and Brunner.

Davanloo, H. (1990). The technique of unlocking the unconscious in patients suffering from functional disorders. Part I. Restructuring ego's defenses. In H. Davanloo (Ed.), Unlocking the unconscious (pp. 283-306). Chichester: John Wiley. 
Davanloo, H. (2000). Intensive short-term dynamic psychotherapy. Central dynamic sequence: Phase of pressure. In H. Davanloo (Ed.), Intensive short-term dynamic psychotherapy: Selected papers of Habib Davanloo (pp. 183-208). New York: Wiley.

Davanloo, H. (2005). Intensive short-term dynamic psychotherapy. In H. Kaplan \& B. Sadock (Eds.), Comprehensive textbook of psychiatry (8th ed., Vol. 2, pp. 2628-2652). Philadelphia: Williams and Wilkins.

Derogatis, L. R. (1993). Brief Symptom Inventory: Administration, scoring, and procedures manual (4th ed.). Minneapolis, MN: Pearson.

Derogatis, L. R. \& Spencer, M. S. (1982). The Brief Symptom Inventory (BSI): Administration, scoring, and procedures manual-I. Baltimore, MD: Johns Hopkins University School of Medicine, Clinical Psychometrics Research Unit.

Department of Health. (2012). Liberating the NHS: No decision about me, without me. Retrieved from https://www.gov.uk/government/uploads/system/uploads/attachment_data/file/216980/Li bera ting-the-NHS-No-decision-about-me-without-me-Government-response.pdf

Derogatis, L. R. (1977). SCL-90-R: Administration, scoring and procedures manual. Baltimore, MD: Clinical Psychometric Research.

Driessen, E., Hegelmaier, L. M., Abbass, A. A., Barber, J. P., Dekker, J. J., Van, H. L., Jansma, E. P., Cuijpers, P. (2015). The efficacy of short-term psychodynamic psychotherapy for depression: A meta-analysis update. Clinical Psychology Review, 42, 1-15. DOI: 10.1016/j.cpr.2015.07.004

Ezriel, H. (1952). Notes on Psychoanalytic Group Therapy: II, Psychiatry, 15(2), 119-126. Doi: $10.1080 / 00332747.1952 .11022866$ 
Evans, C., Mellor-Clark, J., Margison, F., Barkham, M., Audin, K., Connell, J., \& McGrath, G. (2000). CORE: Clinical Outcomes in Routine Evaluation. Journal of Mental Health, 9(3), 247-255. http://doi.org/10.1080/jmh.9.3.247.255

Evans, C., Connell, J., Barkham, M., Margison, F., McGrath, G., Mellor-Clark, J., \& Audin, K. (2002). Towards a standardised brief outcome measure: Psychometric properties and utility of the CORE-OM. British Journal of Psychiatry 180(1), 51-60. https://doi.org/10.1192/bjp.180.1.51

Ferenczi, S. \& Rank, O. (1925). The development of psychoanalysis. Washington, DC: Nervous and Mental Disease Publishing Company.

Gilbody, S., Richards, D., Brealey, S., \& Hewitt, C. (2007). Screening for depression in medical settings with the Patient Health Questionnaire (PHQ): a diagnostic meta-analysis. The Journal of General International Medicine, 22(11), 1596-1602. doi: 10.1007/s11606007-0333-y

Hajkowski, S. \& Buller, S. (2012). Implementing short-term psychodynamic psychotherapy in a tier 4 pathfinder service. Interim report: Derbyshire Healthcare NHS Foundation Trust.

Hinson, V. K., Weinstein, S., Bernard, B., Leurgans, S. E., \& Goetz, C. G. (2006). Singleblind clinical trial of psychotherapy for treatment of psychogenic movement disorders. Parkinsonism and Related Disorders, 12(3), 177-180. https://doi.org/10.1016/j.parkreldis.2005.10.006

Jacobson, N. S., Follette, W. C., \& Revenstorf, D. (1984). Psychotherapy outcome research: Methods for reporting variability and evaluating clinical significance. Behavior Therapy 15(4), 336-352. https://doi.org/10.1016/S0005-7894(84)80002-7 
Kenny, J. (2016). Short-Term Psychodynamic Psychotherapy (STPP) for a Severely Performance Anxious Musician: A Case Report. Journal of Psychology \& Psychotherapy, 6(3). DOI: $10.4172 / 2161-0487.1000272$

Killaspy, H., Banerjee, S., King, M., \& Lloyd, M. (2000). Prospective controlled study of psychiatric out-patient non-attendance. British Journal of Psychiatry, 176(2), 160-165. https://doi.org/10.1192/bjp.176.2.160

Kroenke, K. Spitzer, R. L. \& Williams, J. B. W. (2001). The PHQ-9 - Validity of a brief depression severity measure. The Journal of General International Medicine, 16(9), 606613. DOI:10.1046/j.1525-1497.2001.016009606.x

Kroenke, K., Spitzer, R. L., Williams, J. B. W., \& Löwe, B. (2010). The patient health questionnaire somatic, anxiety, and depressive symptom scales: A systematic review. General Hospital Psychiatry, 32(4), 345-359. DOI: 10.1016/j.genhosppsych.2010.03.006.

Leichsenring, F., Rabung, S., \& Leibing, E. (2004). The efficacy of short-term psychodynamic psychotherapy in specific psychiatric disorders: A meta-analysis. Archives of General Psychiatry, 61(12), 1208-1216. doi:10.1001/archpsyc.61.12.1208

Lemma, A., Target, M \& Fonagy, P. (2011). The development of a brief psychodynamic intervention (dynamic interpersonal therapy) and its application to depression: A pilot study. Psychiatry, 74(1), 41-48. DOI: 10.1521/psyc.2011.74.1.41

Lilliengren, P., Johansson, R., Lindqvist, K.M., Mechler, J., \& Andersson, G. (2016). Efficacy of experiential dynamic therapy for psychiatric conditions: A meta-analysis of randomized controlled trials. Psychotherapy, 53(1), 90-104. https://doi.org/10.1037/pst0000024

Lilliengren, P. (2020). Comprehensive compilation of randomized controlled trials (RCTs) involving psychodynamic treatments and interventions. Available from https://www.researchgate.net/publication/317335876_Comprehensive_compilation_of_r 
Short Term Psychodynamic Psychotherapy in the NHS

andomized_controlled_trials_RCTs_involving_psychodynamic_treatments_and_interven tions $11^{\text {th }}$ July 2020.

Malan, D. H. (1979). Individual psychotherapy and the science of psychodynamics. London: Butterworths.

Manea, L., Gilbody, S., \& McMillan, D. (2012). Optimal cut-off score for diagnosing depression with the Patient Health Questionnaire (PHQ-9): A meta-analysis.Canadian Medical Association Journal, 184(3), E191-E196. DOI:10.1503/cmaj.110829

Markowitz, J. C., Svartberg, M., \& Swartz, H. A. (1998). Is IPT time-limited psychodynamic psychotherapy? The Journal of psychotherapy practice and research, 7(3), 185-195. Retrieved from https://www.ncbi.nlm.nih.gov/pmc/journals/1745/

National Institute for Healthcare and Excellence. (2004). Eating disorders: Core interventions in the treatment and management of anorexia nervosa, bulimia nervosa and related eating disorders. Clinical Guideline (GC9). Retrieved from https://www.nice.org.uk/guidance/cg9/evidence/full-guideline-243824221

National Institute for Healthcare and Excellence. (2017). Eating disorders: Recognition and treatment. Clinical Guideline (NG69). Retrieved from https://www.nice.org.uk/guidance/ng69/chapter/Recommendations

National Institute for Healthcare and Excellence. (2018). Depression in adults: Recognition and management. Clinical Guideline (CG90). Retrieved from https://www.nice.org.uk/guidance/cg90/chapter/1-Guidance

Russell, L., Turner, A. C., \& Yates, P. J. (2017). A preliminary evaluation of intensive short-term dynamic psychotherapy within a functional neurological symptoms service. The Neuropsychologist, 4, 25-33. Retrieved from www.bps.org.uk/publications/neuropsychologist 
Shedler, J. (2010). The efficacy of psychodynamic psychotherapy. American Psychologist, 65(2), 98-109. DOI: $10.1037 / \mathrm{a} 0018378$

Solbakken, A. \& Abbass, A. (2013). Effective care of treatment resistant patients in an ISTDP-based inpatient treatment program. Psychiatric Annals, 43(11), 516-522. Doi: 10.3928/00485713-20131105-07

Spitzer, R. L, Kroenke, K., \& Williams, J. B. W. (1999). Validation and utility of a self-report version of PRIME-MD: The PHQ primary care study. The Journal of the American Medical Association, 282(18),1737-44. DOI:10.1001/jama.282.18.1737

Taylor, P. J., Perry, A., Hutton, P., Tan, R., Fisher, N., Focone, C., Griffiths, D., \& Seddon, C. (2018). Cognitive analytic therapy for psychosis: A case series. Psychology and Psychotherapy: Theory, Research and Practice, 92(3), 1-20. https://doi.org/10.1111/papt.12183

Ten Have-de Labije, J., \& Neborsky, R. J. (2012). Mastering intensive short-term dynamic psychotherapy: A roadmap to the unconscious. (1 $\left.{ }^{\text {st }} \mathrm{ed}.\right)$. London, England: Karnac Books.

Town, J. M., Abbass, A., \& Hardy, G. (2011). Short-Term Psychodynamic Psychotherapy for personality disorders: A critical review of randomized controlled trials. Journal of Personality Disorders, 25(6), 723-740. DOI: 10.1521/pedi.2011.25.6.723.

Town, J. M., Abbass, A., Stride, C., Bernier, D. (2017). A randomised controlled trial of intensive short-term dynamic psychotherapy for treatment resistant depression: The Halifax depression study. Journal of Affective Disorders, 214, 15-25. https://doi.org/10.1016/j.jad.2017.02.035

Town, J. M, Abbass, A., Stride, C., Nunes, A., Bernier, D., \& Berrigan, P. (2020). Efficacy and cost-effectiveness of intensive short-term dynamic psychotherapy for treatment resistant depression: 18 Month follow-up of the Halifax depression trial. Journal of Affective Disorders, 273, 1, 194-202. Doi:10.1016/j.jad.2020.04.035 
Wittkampf, K. A., Naeije, L., Schene, A. H., Huyser, J., \& van Weert, H. C. (2007). Diagnostic accuracy of the mood module of the Patient Health Questionnaire: A systematic review. General Hospital Psychiatry, 29(5), 388-395. DOI:10.1016/j.genhosppsych.2007.06.004 
Short Term Psychodynamic Psychotherapy in the NHS

Tables

Table 1. Summary of Demographic Information

\begin{tabular}{|c|c|c|c|c|c|c|c|}
\hline Participant ID & Age & Gender & Ethnicity & Relationship Status & Education & Employment & Receiving Benefits \\
\hline Participant 1 & 53 & Male & White British & Married & GCSEs & Part-Time & $\mathrm{N}$ \\
\hline Participant 2 & 43 & Male & White British & Married & GCSEs & Full Time & $\mathrm{N}$ \\
\hline Participant $3^{\mathrm{a}}$ & 26 & Female & White British & Married & University & Student & $\mathrm{N}$ \\
\hline Participant 4 & 32 & Female & White British & In a Relationship & A Levels & Part-Time & $\mathrm{Y}$ \\
\hline Participant 5 & 41 & Female & White British & Single & College & Unemployed & $\mathrm{Y}$ \\
\hline Participant 6 & 26 & Female & White British & Divorced & University & Full-Time & $\mathrm{N}$ \\
\hline Participant 7 & 57 & Male & White British & Divorced & College & Unemployed & $\mathrm{Y}$ \\
\hline Participant 8 & 40 & Male & White British & Single & College & Unemployed & Y \\
\hline
\end{tabular}

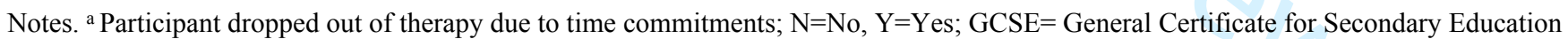


Short Term Psychodynamic Psychotherapy in the NHS

Table 2. Summary of Clinical Information

\begin{tabular}{|c|c|c|c|c|c|c|c|c|}
\hline Participant Id & Diagnosis & Medication & $\begin{array}{c}\text { Previous Suicide } \\
\text { Attempt }\end{array}$ & $\begin{array}{c}\text { Current Self- } \\
\text { Harm }\end{array}$ & Previous Therapy & $\begin{array}{c}\text { Years Under Secondary } \\
\text { MH Support }\end{array}$ & $\begin{array}{c}\text { Sessions } \\
\text { attended/arranged }\end{array}$ & $\begin{array}{c}\text { Completed } \\
\text { Therapy }\end{array}$ \\
\hline Participant 1 & Depression & Venlafaxine & $\mathrm{Y}$ & $\mathrm{N}$ & $>2 \mathrm{x}$ CBT & 1 & $20 / 24$ & Y \\
\hline Participant 2 & $\mathrm{GAD}^{\mathrm{a}}$ & N/A & $\mathrm{Y}$ & $\mathrm{N}$ & $>2 \times \mathrm{CBT}$ & 1 & $20 / 20$ & $\mathrm{Y}$ \\
\hline Participant 3 & $\mathrm{BPD}^{\mathrm{b}}$ & $\begin{array}{l}\text { Quetiapine, paroxetine, } \\
\text { fluoxetine }\end{array}$ & $\mathrm{Y}$ & $\mathrm{N}$ & $>2 \mathrm{x}$ CBT \& $1 \mathrm{DBT}$ & 6 & $4 / 20$ & $\mathrm{~N}$ \\
\hline Participant 4 & $\mathrm{PTSD}^{\mathrm{c}}$ & Quetiapine & $\mathrm{Y}$ & $\mathrm{N}$ & $>2 \mathrm{x}$ CBT \& 1 EMDR & 2 & $17 / 22$ & $\mathrm{Y}$ \\
\hline Participant 5 & BPD & Lamotrogine, diazepam & $\mathrm{N}$ & $\mathrm{N}$ & 1 Counselling & 4 & $14 / 22$ & $\mathrm{Y}$ \\
\hline Participant 6 & Depression & $\begin{array}{l}\text { Venlafaxine, } \\
\text { propanolol, trazodone }\end{array}$ & $\mathrm{N}$ & $\mathrm{Y}$ & $>2 \mathrm{x} \mathrm{CBT}$ & 2 & $19 / 20$ & $\mathrm{Y}$ \\
\hline Participant 7 & Depression & $\begin{array}{l}\text { Olanzapine, } \\
\text { venlafaxine, } \\
\text { mirtazapine }\end{array}$ & $\mathrm{Y}$ & $\mathrm{N}$ & $\begin{array}{l}1 \text { CBT \& } 1 \\
\text { Counselling }\end{array}$ & 4 & $15 / 18$ & $\mathrm{Y}$ \\
\hline Participant 8 & $\begin{array}{l}\text { Paranoid } \\
\text { PD }\end{array}$ & $\begin{array}{l}\text { Aripriprazole, } \\
\text { scitalopram }\end{array}$ & Y & $\mathrm{N}$ & $\begin{array}{l}1 \text { CBT \& } 1 \\
\text { Counselling }\end{array}$ & 13 & $18 / 20$ & Y \\
\hline
\end{tabular}

Notes. ${ }^{\mathrm{a}} \mathrm{GAD}=$ Generalized Anxiety Disorder; ${ }^{\mathrm{b}} \mathrm{BPD}=$ Borderline Personality Disorder; ${ }^{\mathrm{c}} \mathrm{PTSD}=$ Post Traumatic Stress Disorder; ${ }^{\mathrm{d}} \mathrm{PD}=\mathrm{Personality}$ Disorder; $\mathrm{N}=\mathrm{No}, \mathrm{Y}=\mathrm{Y}$ es 
Short Term Psychodynamic Psychotherapy in the NHS

Table 3. Descriptive Statistics and Mean Scores

\begin{tabular}{|c|c|c|c|c|}
\hline \multirow[b]{2}{*}{ Outcome Measure } & \multicolumn{2}{|c|}{ Baseline (Pre-Therapy) Assessment $(\mathrm{N}=8)$} & \multicolumn{2}{|c|}{ Post-Therapy Assessment (N=8) } \\
\hline & $\mathbf{M}$ & SD & $\mathbf{M}$ & SD \\
\hline CORE Wellbeing & 24.06 & 7.89 & 19.68 & 10.89 \\
\hline CORE Symptoms & 22.8 & 8.62 & 16.62 & 8.42 \\
\hline CORE Functioning & 18.12 & 2.64 & 12.87 & 9.16 \\
\hline CORE Risk & 4.96 & 6.2 & 4.13 & 5.75 \\
\hline CORE-OM Total & 18.11 & 3.77 & 13.45 & 7.33 \\
\hline $\mathrm{BSI}^{\mathrm{a}}(\mathrm{GSI})$ & 1.75 & 0.60 & 1.07 & 0.61 \\
\hline PHQ-9 b & 16.00 & 5.23 & 9.63 & 5.18 \\
\hline
\end{tabular}

Notes. ${ }^{\text {a }}$ BSI= Brief Symptom Inventory, GSI=Global Severity Index; ${ }^{\mathrm{b}}$ PHQ-9=Patient Health Questionnaire 


\section{Appendix 1}

\section{Departures from Protocol}

- A recruitment target of $n=6$ was originally planned. However, given that several patients showed an interest in participating in the study, it was increased to $n=8$.

- Initially only pre-post outcome measures were planned to be collected. However, during the study it was decided to add a follow-up measure 8 weeks after the treatment had finished. This was first approved by the Research and Development Department of the Trust and further consent was gained from participants.

- The pre-registered protocol did not include operational definitions of Reliable Change Index (RCI) or the clinically reliable change and this was developed later on.

- The therapy was originally planned to be 1 session/week. However, given that the clinical lead and therapist of the study changed his professional position, he offered 2 sessions during the last week of the treatment to 6 patients and one patient had 2 sessions/week for the last 4 weeks of treatment. The reason for this was to honour the therapeutic agreement with the patients and so that they could complete the full extent of the treatment (i.e. the number of sessions initially agreed). 


\section{Appendix 2}

\section{Statement on Usual Care}

All participants were recruited from a secondary care community mental health team in England. All of them received the usual care from the community team. Usual care involved different types of interventions, following local and national guidelines including: medication, care coordination, peer support, psychiatric review, support worker etc. However, during the study participants were not receiving any other formal psychological intervention (such as CAT, CBT etc.) 


\section{Appendix 3}

\section{Item-by-Item Descriptive Statistics for the Adverse Experiences in Psychotherapy (AEP) Scale at Post-Treatment and 8 weeks Follow-Up}

\begin{tabular}{|c|c|c|c|}
\hline & Item & $\begin{array}{l}\text { Post-Treatment } \\
\text { Mean }(\mathrm{SD})^{\mathrm{a}}\end{array}$ & $\begin{array}{l}8 \text { weeks Mean } \\
(\mathrm{SD})^{\mathrm{b}}\end{array}$ \\
\hline 1. & Taking part hasn't helped me with my problems. & $1.75(1.16)$ & $1.75(1.51)$ \\
\hline 2. & Taking part made my problems worse. & $1.5(0.75)$ & $1.0(0.0)$ \\
\hline 3. & Taking part made me feel more anxious. & $2.0(0.92)$ & $1.75(0.50)$ \\
\hline 4. & Taking part took up too much time. & $1.37(0.74)$ & $1.0(0.0)$ \\
\hline 5. & Taking part led to my mood becoming very low. & $1.37(0.51)$ & $1.75(0.50)$ \\
\hline 6. & Taking part made me feel more angry and irritable. & $1.00(0.00)$ & $1.25(0.50)$ \\
\hline 7. & I didn't feel ready to talk about my problems. & $1.25(0.71)$ & $1.50(1.0)$ \\
\hline 8. & $\begin{array}{l}\text { Taking part made me think too much about bad things that } \\
\text { have happened in the past. }\end{array}$ & $1.87(1.12)$ & $1.50(0.57)$ \\
\hline 9. & Taking part meant I stopped looking after myself properly. & $1.25(0.46)$ & $1.00(0.00)$ \\
\hline 10. & Taking part made me feel more suspicious. & $1.25(0.46)$ & $1.50(1.0)$ \\
\hline 11. & Taking part required too much energy or motivation. & $1.25(0.46)$ & $1.25(0.50)$ \\
\hline 12. & Taking part increased my thoughts of killing myself. & $1.00(0.00)$ & $1.25(0.50)$ \\
\hline 13. & I didn't feel listened to or believed by care staff. & $1.25(0.70)$ & $1.00(0.00)$ \\
\hline 14. & Taking part made my voices or visions worse. & $1.12(0.35)$ & $1.00(0.00)$ \\
\hline 15. & $\begin{array}{l}\text { Taking part was making me fall out with my family or } \\
\text { friends. }\end{array}$ & $1.00(0.00)$ & $1.00(0.00)$ \\
\hline 16. & Taking part was having a bad effect on my self-esteem. & $1.25(0.70)$ & $1.25(0.50)$ \\
\hline 17. & Taking part was making me want to harm myself. & $1.37(0.74)$ & $1.00(0.00)$ \\
\hline 18. & I didn't like or feel I could trust my care team. & $1.25(0.46)$ & $1.00(0.00)$ \\
\hline 19. & $\begin{array}{l}\text { I felt embarrassed talking about my problems with people I } \\
\text { had not met before. }\end{array}$ & $2.25(0.70)$ & $2.75(1.25)$ \\
\hline 20. & $\begin{array}{l}\text { Taking part made me have thoughts of harming other } \\
\text { people. }\end{array}$ & $1.12(0.35)$ & $1.00(0.00)$ \\
\hline 21. & Taking part was making me feel hopeless about the future. & $1.00(0.00)$ & $1.00(0.00)$ \\
\hline 22. & $\begin{array}{l}\text { Taking part meant I had to increase my medication in order } \\
\text { to cope. }\end{array}$ & $1.00(0.00)$ & $1.00(0.00)$ \\
\hline 23. & Taking part involved too much hard work. & $1.00(0.00)$ & $1.00(0.00)$ \\
\hline 24. & Taking part made me worry that people would think badly & $1.37(0.74)$ & $2.25(0.95)$ \\
\hline
\end{tabular}


of me because of my diagnosis.

25. Taking part made me fall out with my doctor or care team. $\quad 1.25(0.70) \quad 1.00(0.00)$

26. Taking part made me worry about losing control of my $\quad 1.00(0.00) \quad 1.00(0.00)$ mind.

27. My problems have improved to the point whereby I no $\quad 2.5(1.51) \quad 3.00$ (1.82) longer feel I need help.

$\mathrm{a} \mathrm{N}=8,{ }^{\mathrm{b}} \mathrm{N}=4$ 
Figures

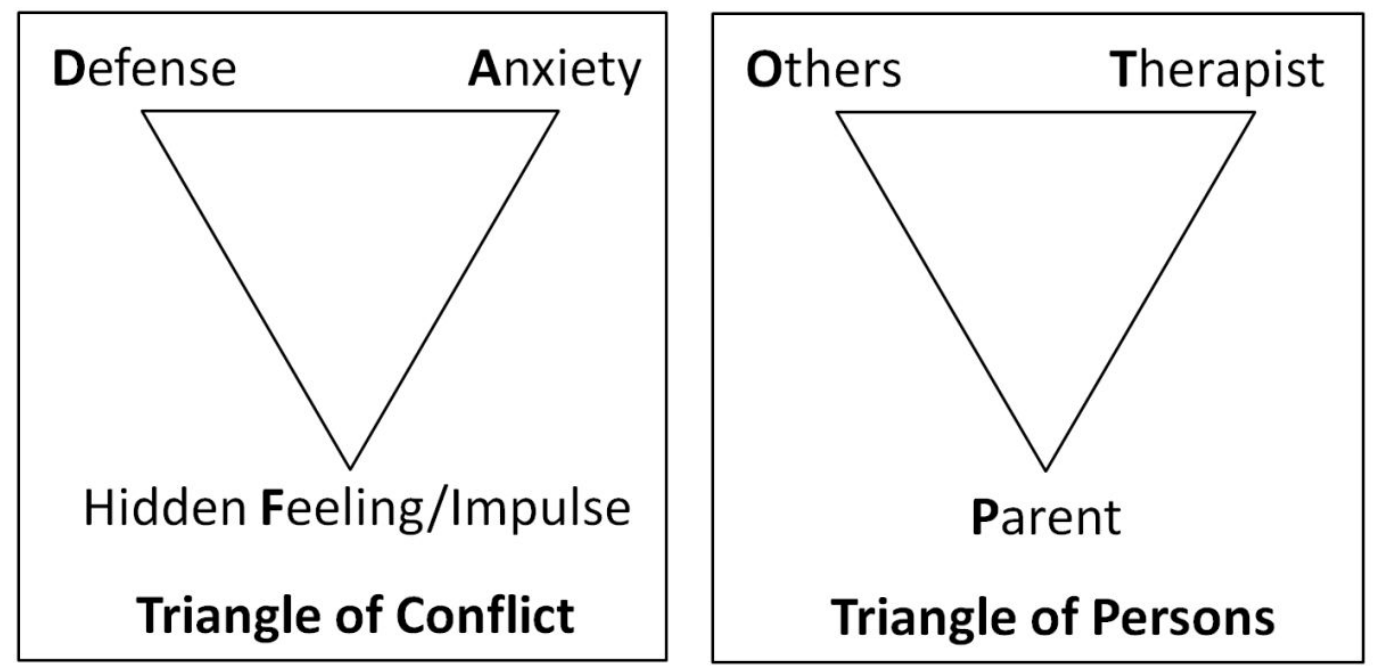

Figure 1: Malan's Triangle of Conflict and Triangle of Persons 


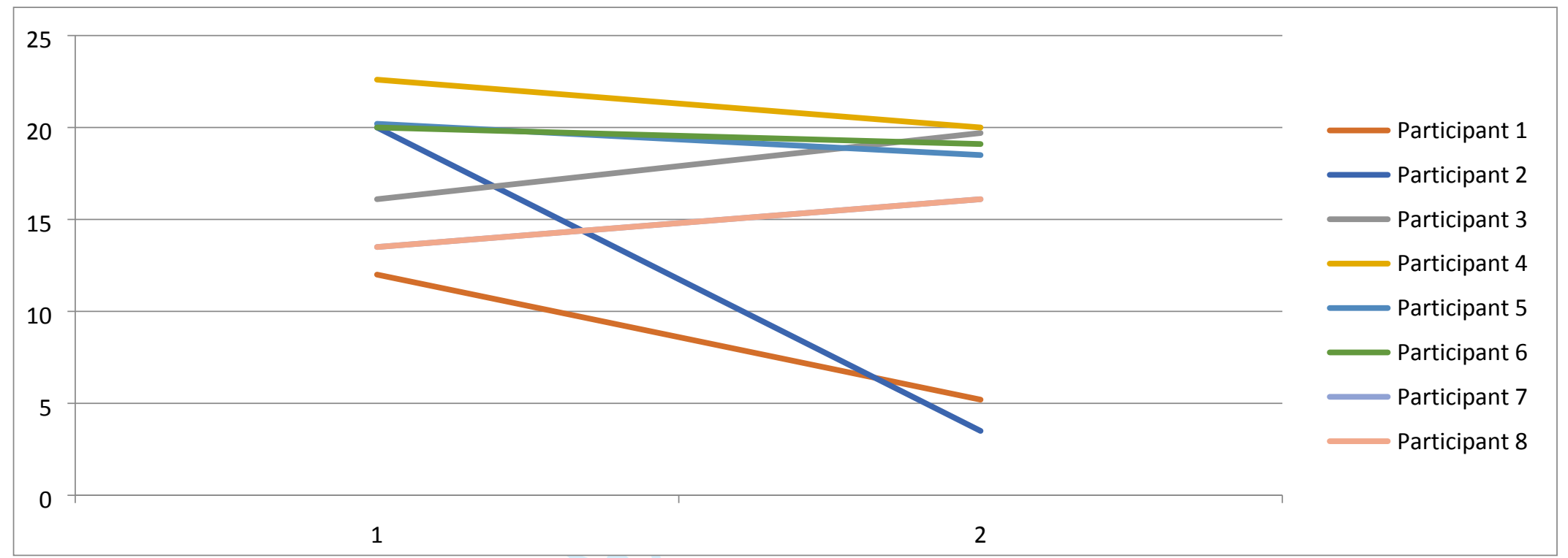

Figure 2a. CORE-OM Pre \& Post Scores

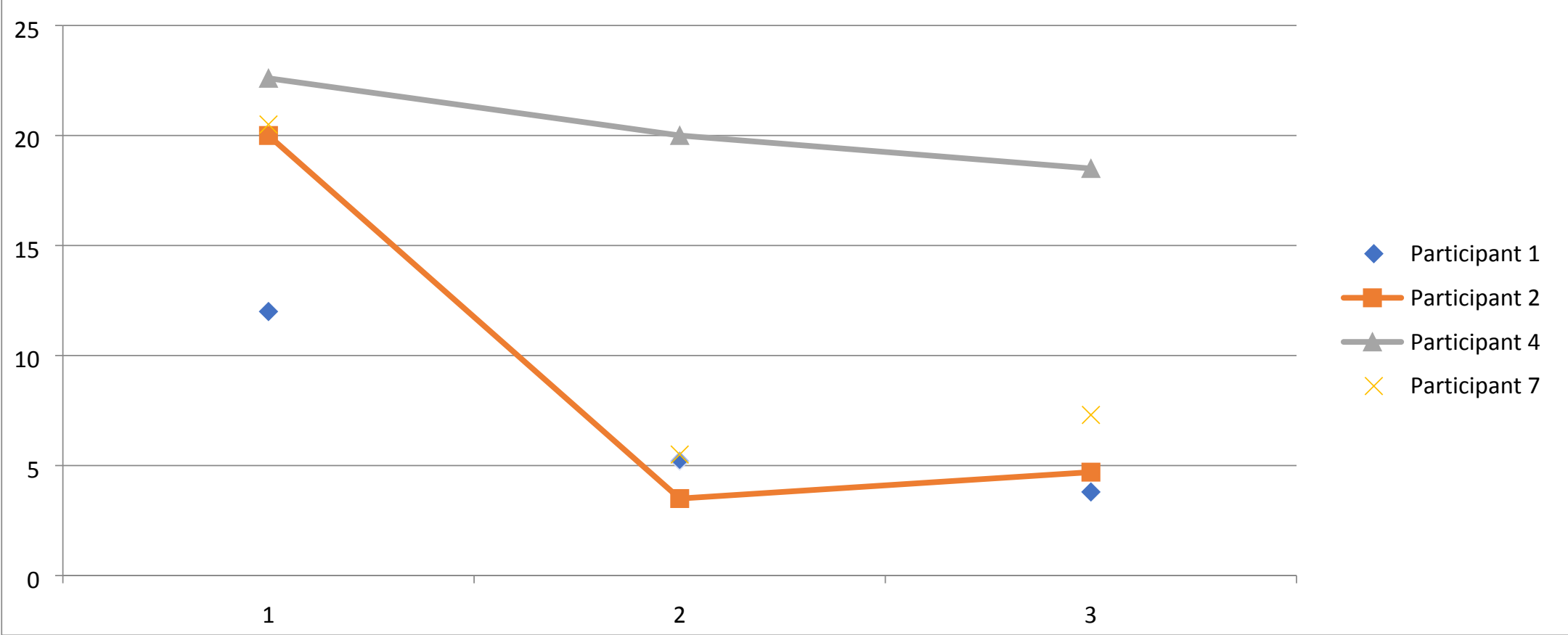

Figure 2b. CORE-OM Pre, Post Scores \& 8 week-follow up scores 


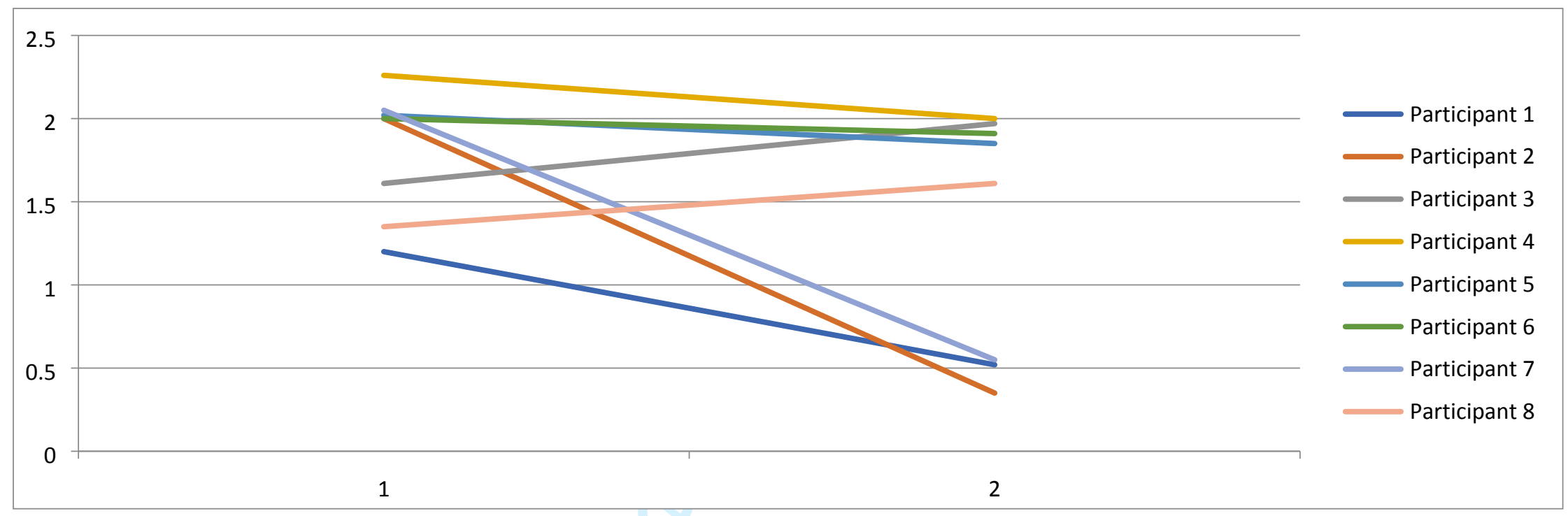

Figure 3a. BSI Pre \& Post Scores

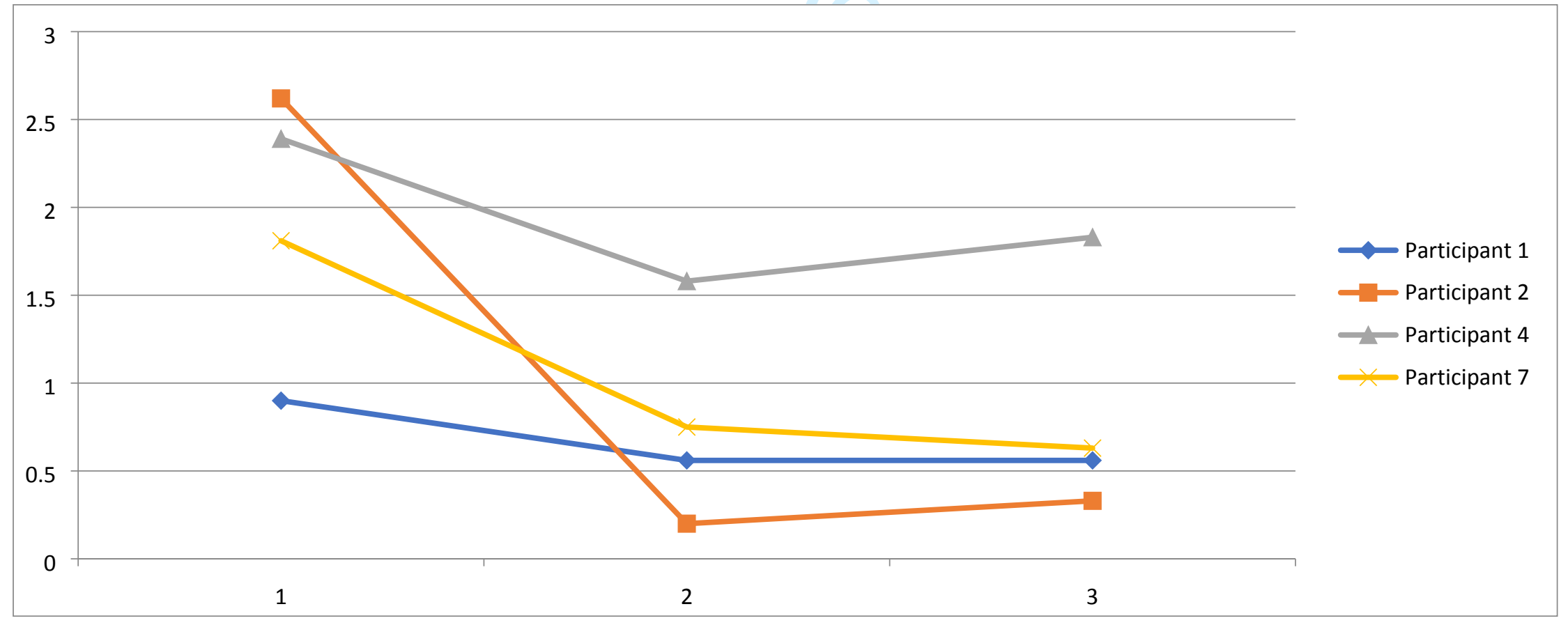

Figure 3b. BSI Pre, Post Scores \& 8 week-follow up scores 


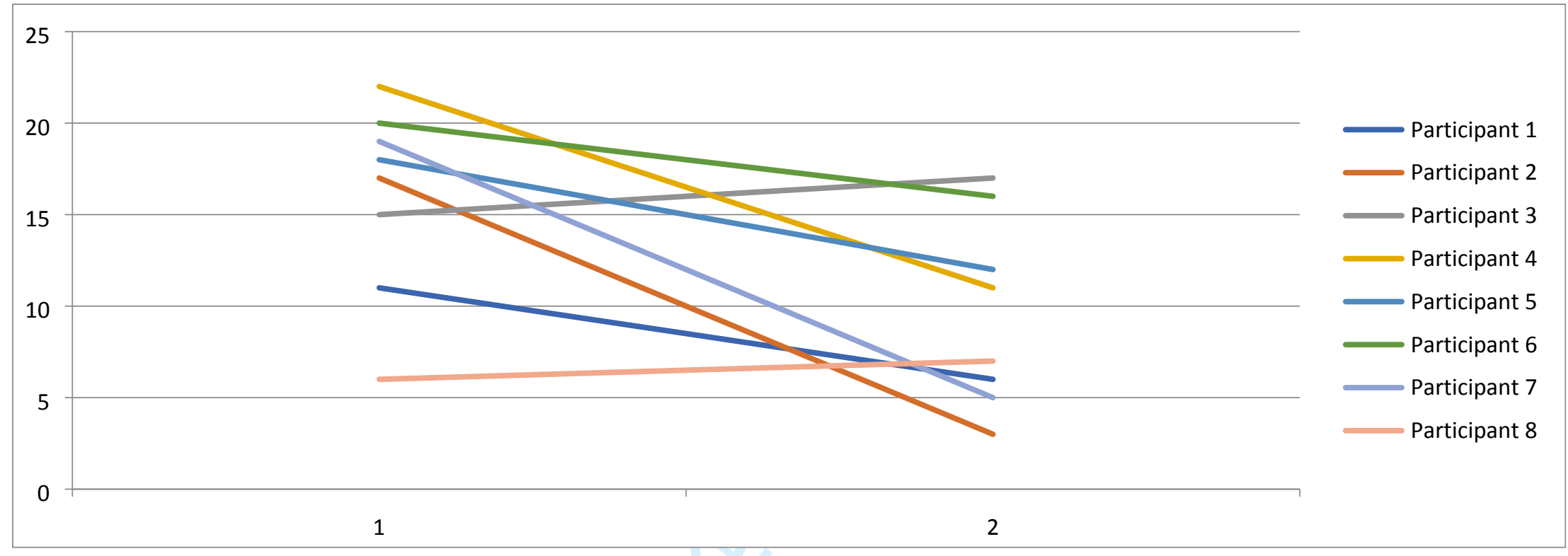

Figure 4a. PHQ-9 Pre \& Post Scores

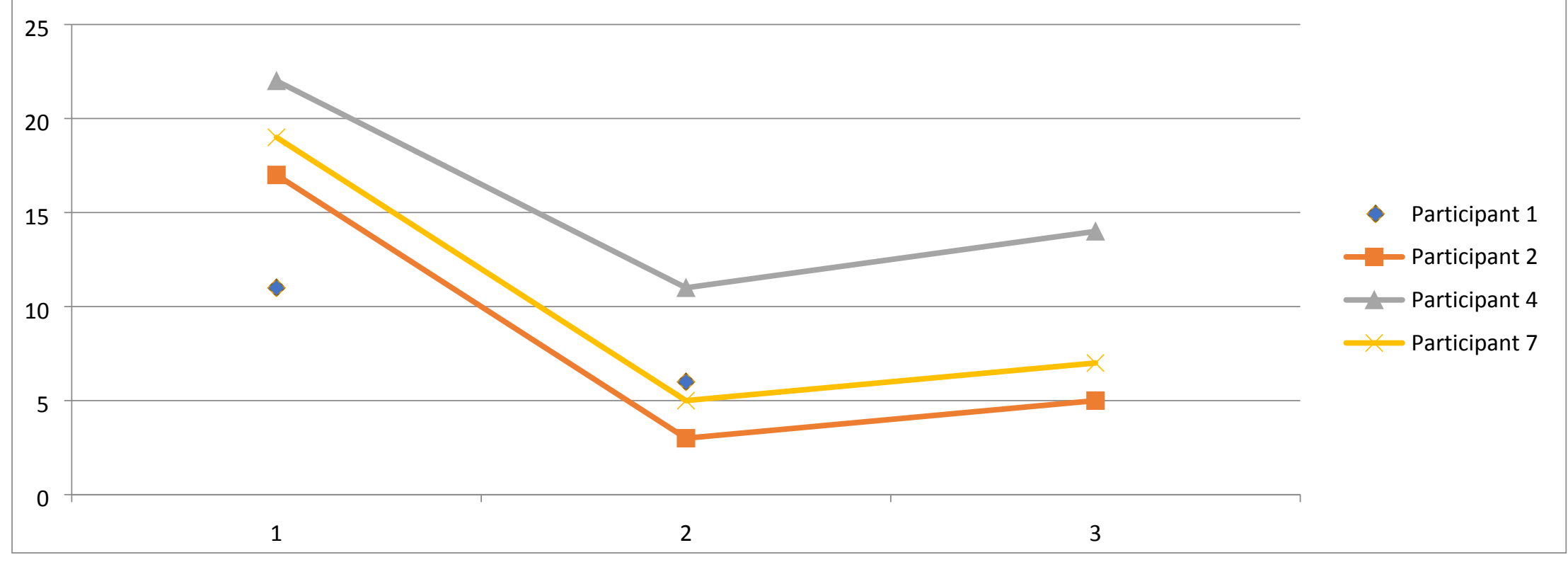

Figure 4b. PHQ-9 Pre, Post Scores \& 8 week-follow up scores 